\title{
Flow Fields within Jupiter's Great Red Spot and White Oval BC
}

\author{
Jim L. Mitchell, ${ }^{1}$ Reta F. Beebe, ${ }^{2}$ ANDRew P. Ingersoll, ${ }^{3}$ AND GlenN W. Garneau ${ }^{4}$ \\ Using sequences of Voyager 1 high-resolution images of Jupiter's Great Red Spot (GRS) and White \\ Oval BC we map the flow fields within the GRS and Oval BC. We compute relative vorticity within these \\ features as a function of semi-major axis length and position angle in a coordinate system consisting of \\ concentric ellipses of equal eccentricity. Both the velocity and the relative vorticity profiles are nearly \\ identical for Oval BC and the outer portion of the GRS. Wind speeds of 110-120 m/s are observed near \\ the outer edges of both features. Along their minor axes relative vorticity profiles reach a maximum of \\ $\sim 6 \times 10^{-5} \mathrm{~s}^{-1}$. This is several times greater than the ambient $1.5 \times 10^{-5} \mathrm{~s}^{-1}$ meridional shear of zonal \\ winds at the latitudes of the GRS and Oval BC. Maximum Rossby numbers of 0.36 are computed for \\ flows within both the GRS and the Oval BC. Generally, the Rossby numbers within these features are \\ much lower, indicating strongly geostrophic constraints on the flow. The difference in streamline curva- \\ ture within the GRS and the Oval BC is found to compensate for the difference in planetary vorticity at \\ the respective latitudes of the features. Motions within the central region of the GRS are much slower \\ and more random than around the spot's outer portion.
}

\section{INTRODUCTION}

Jupiter's Great Red Spot (GRS) has attracted much popular and scientific interest over the past century. During that time a host of theories have been put forward to fill what remained a noticeable observational void. Though careful records were kept of the color, size, shape, and longitude of the GRS, observations of smaller spots moving anticyclonically around the periphery of the GRS [Reese and Smith, 1968] remained the only observation of the nature of the flow field of the GRS. The flow fields around and within the smaller White Ovals which began to form about 40 years ago at $34^{\circ} \mathrm{S}$ remained totally unobservable from earth. In the following study we report on the use of sequences of high resolution Voyager images to map the flow fields within the GRS and one of the three White Ovals, which referring to standard nomenclature [Peek, 1958] we call Oval BC.

Before continuing with a presentation of our measurements we provide a brief description of the cloud morphologies within the GRS and Oval BC. As was noted earlier [Mitchell et al., 1979], there seems to be a general lack of,organized cloud structure in the mottled, innermost portion of the GRS and to a lesser extent the White Ovals (suggestive of low speed flows and perhaps convective activity). Referring to Figures 1 (top) and 2 (top), one sees that the lack of organization in the spot's interior gives way to what appears to be a slightly unwinding spiral pattern as one moves outward to the spot's edge. Chevron-shaped cloud patterns around the outer portions of the spot suggest that highest velocities occur near the spot's periphery. Both the GRS and the Oval BC are surrounded by a collar of darker clouds. This morphological description fits both the GRS and the Oval BC, as well as the remaining two White Ovals.

\section{VELOCITY FIELDS}

We used the AMOS Interactive System at JPL's Image Processing Laboratory [Yagi et al., 1978] to measure dis-

\footnotetext{
1 Jet Propulsion Laboratory, California Institute of Technology, Pasadena, California 91103.

2 Department of Astronomy, New Mexico State University, Las Cruces, New Mexico 88003.

${ }^{3}$ Division of Geological and Planetary Science, California Institute of Technology, Pasadena, California 91125.

4 Image Processing Laboratory, Jet Propulsion Laboratory, California Institute of Technology, Pasadena, California 91103.

Copyright $\mathcal{C} 1981$ by the American Geophysical Union.
}

placements of small cloud features (tracers) within the GRS and Oval BC between pairs of Voyager 1 narrow-angle camera images taken through a green filter. Both sets of images were taken on March 4, 1979, and cover the GRS and Oval BC at a spatial resolution of approximately $24 \mathrm{~km} /$ vidicon line pair. The average temporal separation of the GRS pairs is 0.55 hour, resulting in a minimum resolved velocity of $12 \mathrm{~m} / \mathrm{s}$; while the average temporal separation of the Oval BC pairs is 1.39 hour, resulting in a minimum resolved velocity of $5 \mathrm{~m} / \mathrm{s}$. Normally we would convert a tracer's location in the Voyager image matrix of picture elements (pixels) to a corresponding point in jovian latitude and longitude by fitting the limb of a map grid to the actual jovian limb as seen in a simultaneously shuttered wide-angle image. In the case of those GRS and Oval BC images, upon which we base this report, the spacecraft was too close to the planet for the limb to be observable in the wide-angle image. In such a case tracers with velocities measured from earlier image pairs (containing a planetary limb in the associated wide-angle image) are located in the higher resolution image pairs and defined to have the same velocity as was earlier measured. On the basis of several such common tracers, a map grid may then be fit to the high resolution image pairs. Though such a method enhances corresponding errors in measured absolute velocities, relative velocities within a pair of frames remains reliable. We have compared the high resolution GRS velocity field with a similar field measured from a pair of image sets taken 10 and 20 hours earlier. The wide-angle camera field of view included the limb in the earliest set of images (i.e., that taken 20 hours earlier). The velocity field as measured from the earlier pair does not seem to significantly differ from the field measured in the later, higher resolution set. This earlier set of pairs with a temporal separation of 10 hours yielded a sparser field of tracers because of the difficulty involved in trying to identify individual tracers over a 10 hour interval.

As seen in Figures 1 (bottom) and 2 (bottom), the tracers used to measure the velocity fields are not uniformly spaced in a grid of latitude and longitude. In the case of the GRS the fields of view do not cover the outermost portions of the GRS along the spot's minor axis. As it is the chief objective of this report to present several observed characteristics of the flow fields within the GRS and Oval BC for future comparison with theory, we fit our observed velocities to a smooth function of distance from the center of the spots. In order to do this we overlay on the GRS and Oval BC a grid of concentric 

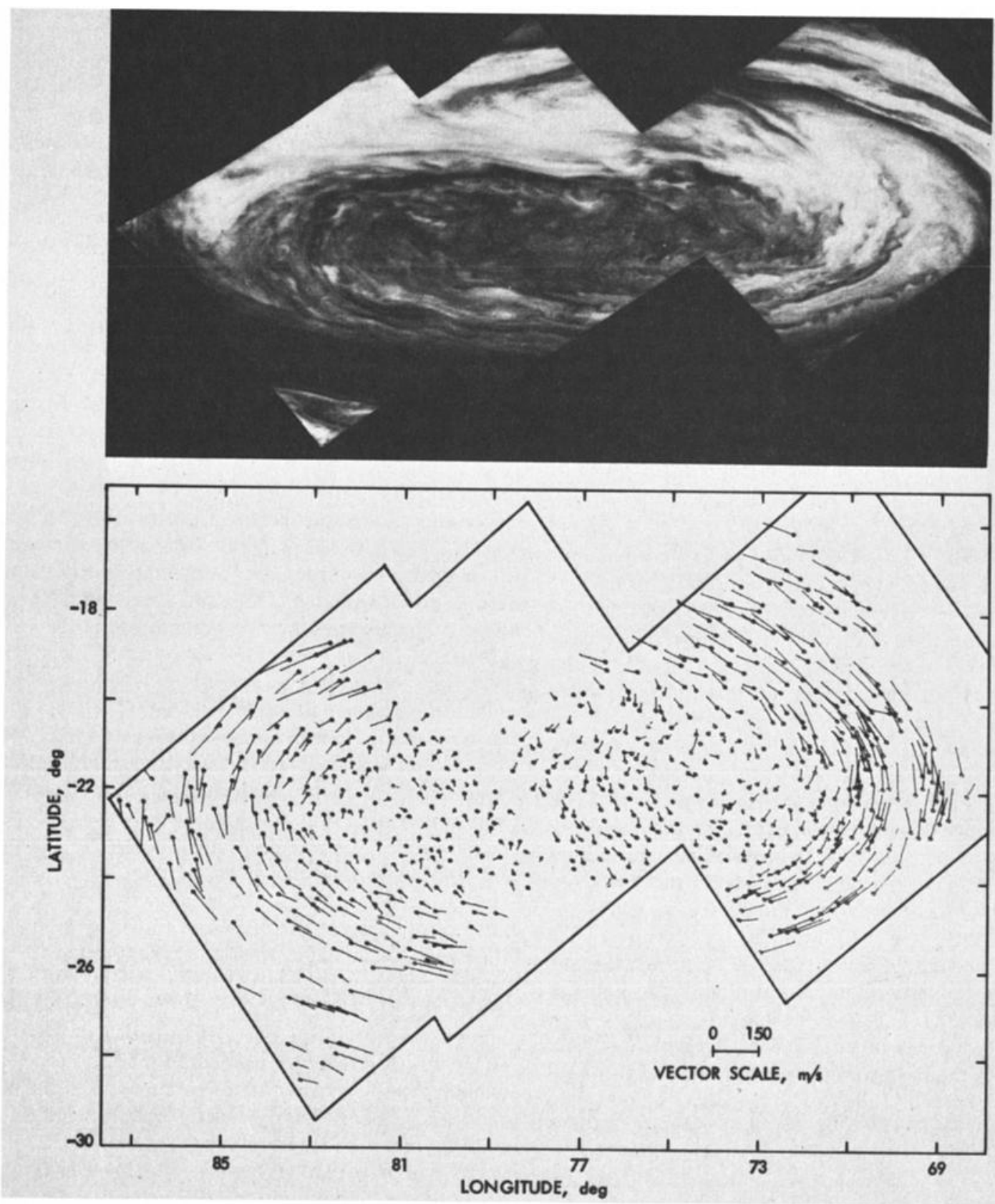

Fig. 1. (Top) Great Red Spot mosaic of three green narrow-angle camera images taken on March 4, 1979, by Voyager 1. Spatial resolution in the original images is $24 \mathrm{~km} /$ vidicon line pair. (Bottom) Flow field within the GRS. The above images and others taken an average of 0.55 hours later were used to measure motions of small cloud tracers within the GRS. Circle indicates earlier location of tracer. Length of arrow is proportional to wind speed, while arrow points downwind. Latitude is planetographic, while longitude is system III (1965.0).

ellipses of constant eccentricity ( $\varepsilon$ ). The outermost ellipse (i.e., that around the dark peripheral collar of each feature) is found to have the dimensions provided in Table 1 where associated eccentricities are also given. For an illustration of the geometry of concentric ellipses, refer to Figure 3. We find that the velocity at a point within the GRS or Oval BC is for the most part in a direction tangent to that ellipse passing through the point. Hence, we ignore the small radial velocity component in the following treatment.

In the interior of both the GRS and Oval BC the tangential velocities are not strongly $\theta$ dependent. We express the tangential velocity as a function of the semi-major axis length $(a)$ of the particular ellipse upon which the point lies. We fit the observed velocities to a fourth order polynomial in a given by

$$
V_{\mathrm{T}}(a)=C_{1} a+C_{2} a^{2}+C_{3} a^{3}+C_{4} a^{4}
$$

A least squares fit to (1) gives the coefficient values provided in Table 2. The actual velocity profiles within the GRS and Oval BC are provided as a function of $a$ in Figures 4 and 5 , respectively.

The quiescent nature of the flow field in the GRS out to about $a=4.2 \times 10^{6} \mathrm{~m}$ is readily apparent in Figure 4 . We note that the smoothed tangential velocity reaches a sharp maximum of about $110 \mathrm{~m} / \mathrm{s}$ at $a=10.2 \times 10^{6} \mathrm{~m}$ (about $85 \%$ of the distance out from the center to the edge of the GRS). The smoothed tangential velocity within Oval BC reaches a similar maximum near the outer edge of the feature. Though not shown in Figure 5, the velocities just outside Oval BC drop off 


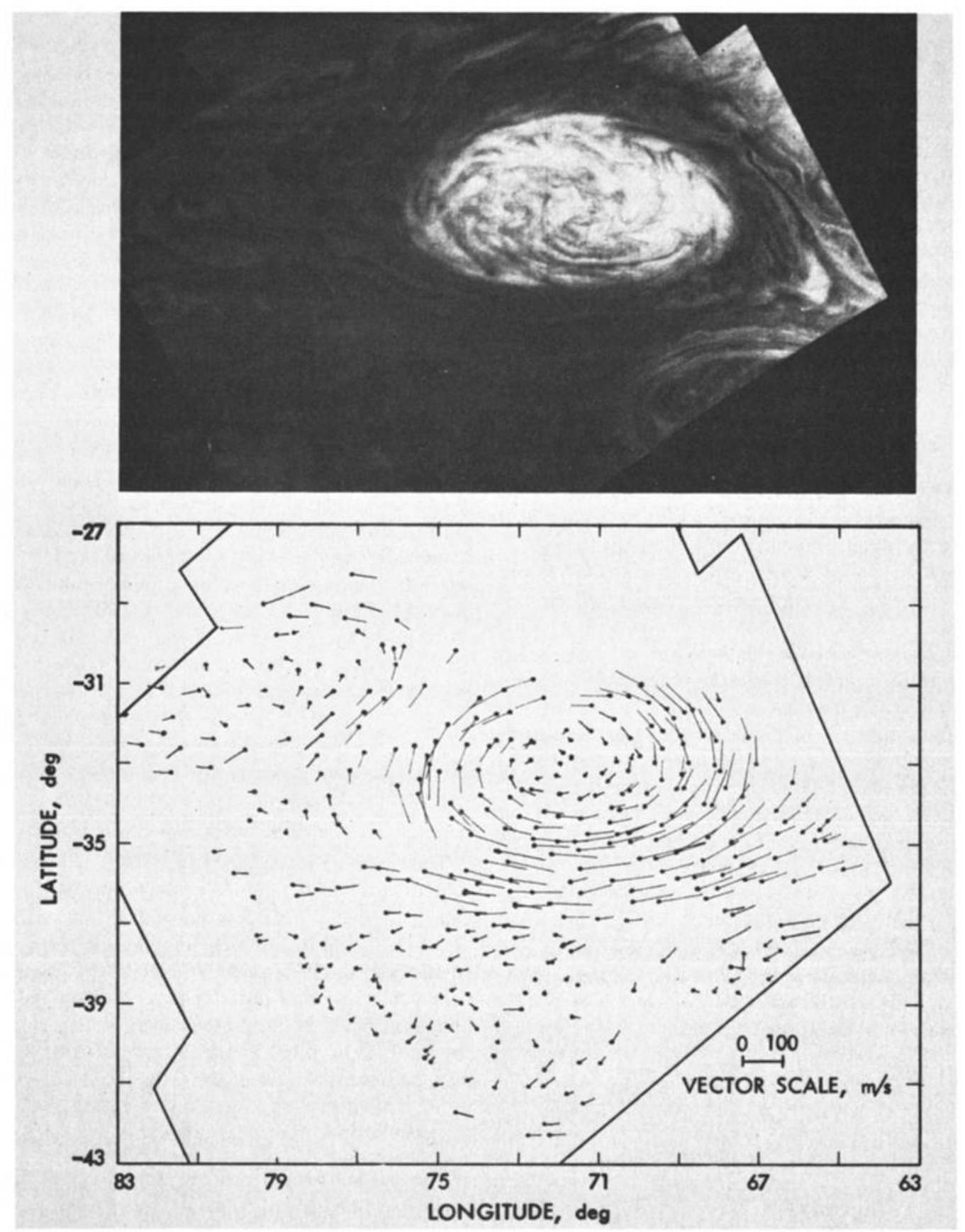

Fig. 2. (Top) Oval BC mosaic of four green narrow-angle camorn images taken on March 4, 1979, by Voyager 1. Spatial resolution in the original images is $24 \mathrm{~km} /$ vidicon line pair. (Bottom) Flow field within Oval BC. The above images and others taken 1.39 hours later were used to measure motions of small cloud tracers within Oval BC. Circle indicates earlier location of tracer. Length of arrow is proportional to wind speed, while arrow points downwind. Latitude is planetographic, while longitude is system III (1965.0).

rapidly with increasing a just as for the GRS. For all purposes the velocity profile within Oval $B C$ is identical to the velocity profile in the GRS from $a=4.2 \times 10^{6} \mathrm{~m}$ outward. The velocities we report for Oval BC are higher than those implied earlier [Smith et al., 1979]. Earlier reports were based upon image pairs temporally separated by 10 hours or more. Our 1.39 hour temporal separation allowed the identification of small tracers within the high speed peripheral jet, leading to the measurement of higher velocities.

\section{VORTICITY}

For flow along streamlines consisting of concentric ellipses the relative vorticity $(\zeta$ may be expressed as (see appendix)

$$
\zeta(a, \theta)=\frac{a}{b^{2} \eta^{3}} V_{T}+\eta \frac{d V}{d a} T
$$

where,

$$
\eta=\left[\frac{\cos ^{2} \theta+\left(\frac{a}{b}\right)^{4} \sin ^{2} \theta}{\cos ^{2} \theta+\left(\frac{a}{b}\right)^{2} \sin ^{2} \theta}\right]^{1 / 2}
$$

$V_{T}=$ velocity tangent to ellipse of semi-major axis $a ; a, b, \theta$ are as shown in Figure 3. With $V_{T}(a)$ expressed by (1) we are thus able to compute $\zeta(a, \theta)$. Plots of the relative vorticity 
TABLE 1. Outer Dimensions of GRS and Oval BC

\begin{tabular}{lccc}
\hline & $\begin{array}{c}\text { Semi-major } \\
\text { Axis }(a)\end{array}$ & $\begin{array}{c}\text { Semi-minor } \\
\text { Axis }(b)\end{array}$ & $\begin{array}{c}\text { Eccentricity } \\
(\varepsilon)\end{array}$ \\
\hline GRS & $12.08 \times 10^{6} \mathrm{~m}$ & $5.58 \times 10^{6} \mathrm{~m}$ & 0.887 \\
Oval BC & $4.89 \times 10^{6} \mathrm{~m}$ & $2.93 \times 10^{6} \mathrm{~m}$ & 0.801 \\
\hline
\end{tabular}

within the GRS and Oval BC are provided in Figures 6 and 7. Again, we have neglected the radial velocity component, which is $\theta$ dependent, hence the illustrated $\theta$ dependence of relative vorticity is only approximate.

It is interesting to note that the vorticity is concentrated in the outer portions of both features and has the same characteristic profile for Oval BC (though of greater magnitude along the major axis) as for the GRS from $4.2 \times 10^{6} \mathrm{~m}$ outward.

We note that along their minor axes (i.e., $\theta=90^{\circ}$ ) the GRS and Oval BC reach nearly identical vorticity maxima of roughly $6.3 \times 10^{-5} \mathrm{~s}^{-1}$. The vorticity of the ambient shears in which both features sit is approximately $1.5 \times 10^{-5} \mathrm{~s}^{-1}$ [Beebe et al., 1980]. Thus, a key to comparison with theory is the observation that the intrinsic vorticity of the GRS and Oval BC is several times greater than the vorticity of the ambient shear.

It is interesting to compare the relative vorticity of the GRS and Oval BC with the ambient planetary vorticity $f \equiv 2 \Omega$ sin $\phi$, where $\Omega$ is the jovian angular rotational velocity and $\phi$ is the planetographic latitude). In Table 3 we list $f$ at the centers of the GRS $\left(\phi=22^{\circ} .5 \mathrm{~S}\right)$ and Oval BC $\left(\phi=33^{\circ} \mathrm{S}\right)$ as well as the change in $f$ from the spot's southern to northern edge $(\Delta f)$. We also compute a Rossby number $(R o)$ for the spot defined as the absolute value of the ratio of the relative vorticity maximum along the minor axis $(\zeta \max )$ to the planetary vorticity $(f)$ at the latitude of the spot's center. Computed values are 0.45 and 0.33 for the GRS and Oval BC.

Alternatively, the Rossby number $(R o)$ is defined as the ratio of the inertial acceleration to the Coriolis acceleration. For flow in gradient balance the inertial acceleration is purely centrifugal, hence $R o$ is given by

$$
R o=\frac{V_{T} a / b^{2} \eta^{3}}{f}
$$

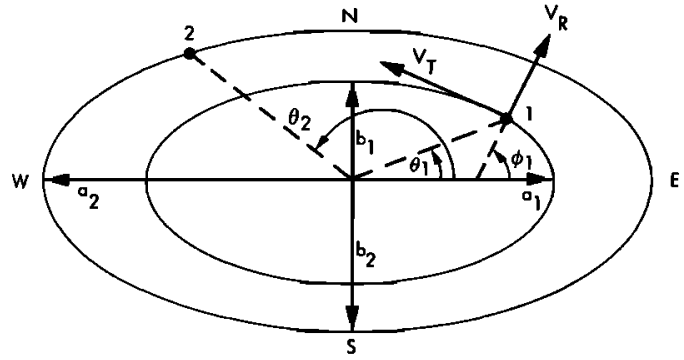

Fig. 3. Geometry of concentric ellipses of equal eccentricity. We fit observed velocities within the GRS and Oval BC to a coordinate system consisting of concentric ellipses. The location of any point within the GRS or Oval BC is defined by specifying the semi-major axis length $(a)$ of the ellipse upon which the point lies and the position angle measured positive counterclockwise from due east ( $(\theta)$. The second position angle $(\phi)$ corresponds to a 'geographic' latitude on an elliptical surface. Since all ellipses are of equal eccentricity the semi-minor axis length $(b)$ is determined by specification of $a$. The tangential velocity component $\left(V_{T}\right)$ is then that component tangent to the perimeter of a given ellipse, while the radial component $\left(V_{R}\right)$ is in the direction normal to that ellipse. Cardinal points of the compass are shown as $\mathbf{E}$ (east), $\mathbf{N}$ (north), etc. where $b^{2} \eta^{3} / a=$ radius of curvature of the flow $(a, b$, and $\eta$ as previously defined). Thus, using our smoothed velocity field, $R o$ is a function of the independent variable $V_{T}, \Theta$, and $\phi$.

We are most interested in the maximum value reached by $R o$ since this indicates locations where the geostrophic constraint is weakest. Ro becomes a maximum along the spot's major axis $\left(\theta=0^{\circ}, 180^{\circ}\right)$ at that location where $V_{T}$ also reaches a maximum ( $\left.V_{T} \max \right)$. For the GRS we take $V_{T} \max$ $=110 \mathrm{~m} / \mathrm{s}\left(\right.$ at $\left.a=10.5 \times 10^{6} \mathrm{~m}\right)$; this gives a maximum Rossby number within the GRS of 0.36 (neglecting the minus sign). Along the minor axis $\left(\theta=90^{\circ}, 270^{\circ}\right)$ the maximum Rossby number has dropped to 0.04 . Thus, along the major axis the flow is in gradient balance, while along the minor axis the flow is in geostrophic balance.

For Oval BC we take $V_{T} \max =120 \mathrm{~m} / \mathrm{s}$ (at $a=4.9 \times 10^{6}$ $\mathrm{m}$ ); this again gives a maximum Rossby number of 0.36 within Oval BC. Along the minor axis the maximum Rossby number has dropped to 0.08 . Thus, the Rossby number of flow within the GRS and Oval BC is nearly the same at equivalent locations within the two features. It is very interesting that the difference in the streamline curvature of the GRS and Oval BC appears to compensate for the difference in latitude of the two features. This could imply that the difference in size of the GRS and Oval BC is related to the difference in their respective latitudes. The low values taken by $R o$ at most locations within these features indicate that geostrophy is a strong constraint on their internal flow except perhaps along their major axes. These results for the GRS agree well with an earlier report based upon earth-based observation [Hess, 1969].

\section{DIVERGENCE/CONVERGENCE}

The smaller radial velocity component is important in any consideration of the divergence or convergence of the GRS or Oval BC. Because we have neglected the radial component, we are not able to comment in detail on the divergence/convergence of the flow field. We believe that divergence or convergence is a second-order effect in the flow fields of the GRS and Oval BC. The fact that smaller eddies, originating along the south edge of the South Equatorial Belt (SEB) are drawn into the outer portions of the GRS [Ingersoll et al., 1979] may be indicative of vortex interaction rather than of a net convergence around the outer portion of the GRS.

\section{Role of Eddies in the Jovian Global Circulation}

What is the direction and magnitude of net momentum and kinetic energy transport between the eddies (e.g., GRS and Oval BC) and the mean zonal flow? This is a key question in the dynamics of any atmosphere. In other reports [Beebe et al., 1980; Ingersoll et al., this issue] we look at the net role of eddy flow in maintaining the mean zonal circulation. We find that the kinetic energy cascades in a negative sense from the eddy motions into the mean flow. In this connection we note that a purely symmetrical (i.e., velocity not a function of $\theta$ ) elliptical eddy with a perfectly zonal major axis will transport

TABLE 2. Least Squares Coefficients for $V_{T}(a)$ for $V_{T}$ given in $\mathrm{m} / \mathrm{s}$ and $a$ in $10^{6} \mathrm{~m}$

\begin{tabular}{ccc}
\hline & GRS & Oval BC \\
\hline$C_{1}$ & 9.664 & 8.334 \\
$C_{2}$ & -7.959 & 4.075 \\
$C_{3}$ & 1.665 & 0.024 \\
$C_{4}$ & -0.0858 & -0.046 \\
\hline
\end{tabular}




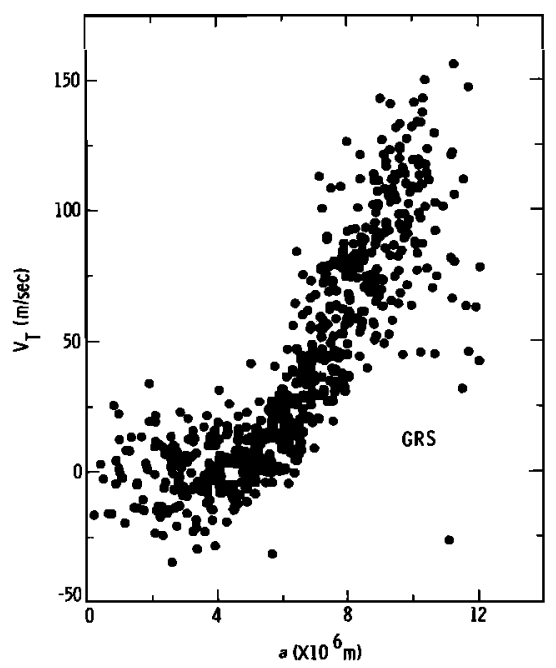

Fig. 4. Observed tangential velocities $\left(V_{T}\right)$ as a function of $a$ within the GRS.

no net kinetic energy into or out of the mean zonal flow. Hence, our smoothed tangential velocity fields for the GRS and Oval BC have no net kinetic energy transport associated with them. We have looked for statistically significant $\theta$ dependencies in the velocity fields or slight tilts in the orientation of the elliptical streamlines but have not yet reached any conclusions. Should searches for such asymmetries be productive, the resulting magnitude and direction of kinetic energy transport will provide an interesting comparison with our associated reports as well as with theory.

\section{COMPARISONS AND CONCLUSIONS}

Dynamically, the similarities between the GRS and Oval $B C$ are indeed striking. If we were to somehow remove the inner portion of the GRS (out to about $a=4.2 \times 10^{6} \mathrm{~m}$ ) and shrink what remained around its center, the internal flow field of the GRS would be nearly identical with the flow field inside Oval BC. We point out that upwelling in the interior of the GRS has often been suggested as being responsible for the characteristic reddish color by the upward transport of red materials from deep within the atmosphere [Khare and Sagan, 1973]. Could it be that the quiescent inner portion of the GRS

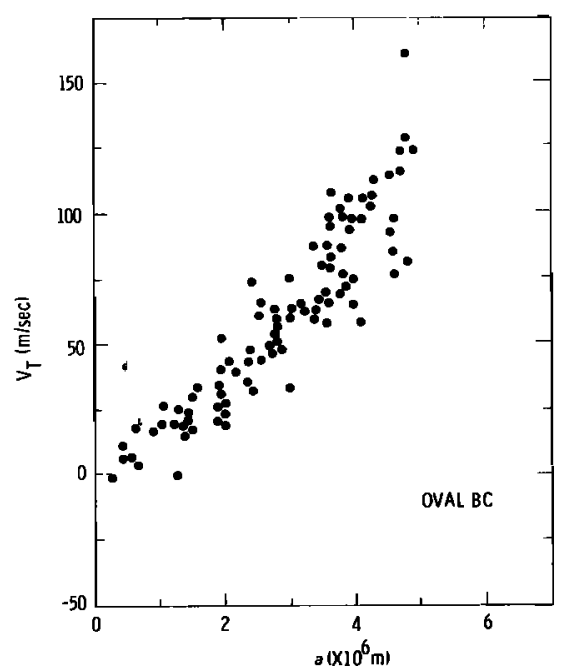

Fig. 5. Observed tangential velocities $\left(V_{T}\right)$ as a function of $a$ within Oval BC.

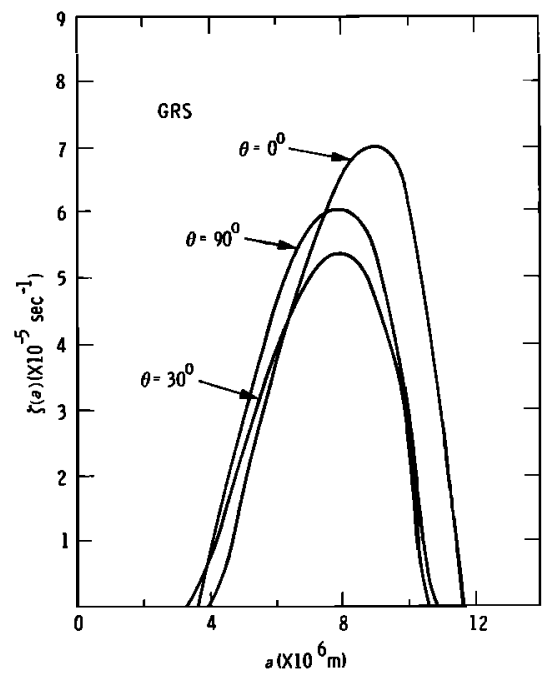

Fig. 6. Relative vorticity $(\zeta)$ as a function of $a$ and $\Theta$ within the GRS.

is indeed such a region of upwelling and that the White Ovals lack red clouds because they also lack such an interior region of upwelling? We have no direct way of observing such vertical motions with any sequence of Voyager images, hence we cannot directly confirm upwelling in the inner portion of the GRS. The observations of lower temperatures in the central regions of the GRS [Hanel et al., 1979a, b] may stupport the notion of central upwelling. However, at spatial resolutions higher than in their published global maps of brightness temperature, the Voyager infrared investigators report a thermal structure for the White Ovals very similar to that of the GRS [Flasar et al., this issue; Conrath et al., this issue]. They interpret this as indicative of upwelling within the White Ovals as well as the GRS.

The Voyager images of the GRS would, on their surface, eliminate a host of models, many of which were already somewhat out of vogue, such as 'floating rafts' [Peek, 1958; Streett et al., 1971]. Though the quiescence of the inner portion of the GRS seems reminiscent of the stagnation of flow within a Taylor-Proudman column, the closed, eliptical streamlines of the outer flow does not reserable flow around such a columa [Hide and Ibbetson, 1966; Ingersol, 1969]. Tests of the remain-

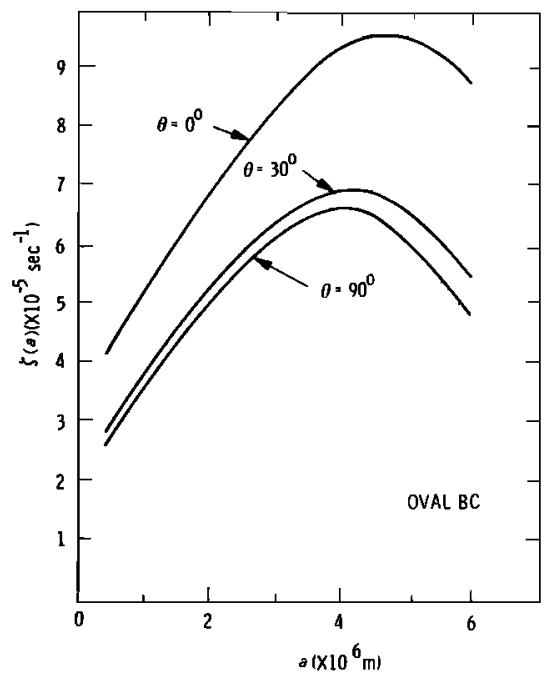

Fig. 7. Relative vorticity $(\zeta)$ as a function of $a$ and $\theta$ within Oval BC. 
TABLE 3. Comparison Between Relative and Planetary Vorticity

\begin{tabular}{lcccc}
\hline & $\begin{array}{c}\zeta \max \\
\left(\times 10^{-5} \mathrm{~s}^{-1}\right)\end{array}$ & $\begin{array}{c}f \text { at centers } \\
\left(\times 10^{-5} \mathrm{~s}^{-1}\right)\end{array}$ & $\begin{array}{c}\Delta f \\
\left(\times 10^{-5} \mathrm{~s}^{-1}\right)\end{array}$ & $R o(\max )$ \\
\hline GRS & 6.1 & -13.5 & 6.0 & 0.36 \\
Oval BC & 6.4 & -19.2 & 2.6 & 0.36 \\
\hline
\end{tabular}

ing GRS hypotheses, such as the notions of a baroclinic eddy [Williams, 1979], a solitary Rossby wave [Maxworthy and Redekopp, 1976], and a free barotropic vortex [Ingersoll, 1973] will need to await planned future studies of the energetics of the GRS and White Ovals.

In closing we note that a truth-table analysis was once suggested for testing GRS models [Sagan, 1971]. To that list of characteristics, which any good model of the GRS (and for the most part any model of the White Ovals) would be called upon to explain, we are able to add the following:

1. Flow within the GRS and Oval BC is for the most part azimuthally symmetrical and along closed, elliptical streamlines.

2. Vorticity is concentrated around the outer portions of the GRS and Oval BC

3. The magnitude of both the velocity and vorticity (along the minor axis) maxima are roughly identical for the GRS and Oval BC. The vorticity maximum greatly exceeds the vorticity of the ambient shear.

4. The difference in streamline curvature for the GRS and Oval BC compensates for their difference in latitude so that Rossby numbers associated with the flow within both features seem to be nearly identical. For the most part the flow is strongly geostrophic.

5. In contrast to the White Ovals, the GRS possesses a large quiescent interior region where velocities are much lower and more random.

\section{APPENDIX: DERIVATION OF EQUATION}

Referenced to Figure 3 and neglecting subscripts on $\phi$ and $\theta$ we may write [Peek, 1958, see Appendix 1]

$$
\tan \phi=\lambda \tan \theta
$$

where

$$
\lambda=\left(\frac{a}{b}\right)^{2}
$$

Let $\tan \theta=y / x$. That is, we set up a Cartesian system with origin at the ellipse center; $x$ runs in the $a$ direction, while $y$ runs in the $b$ direction. Thus,

$$
\begin{aligned}
\sin \phi & =\frac{\lambda y}{\left(x^{2}+\lambda^{2} y^{2}\right)^{1 / 2}} \\
\cos \phi & =\frac{x}{\left(x^{2}+\lambda^{2} y^{2}\right)^{1 / 2}}
\end{aligned}
$$

Hence, if $u$ and $v$ represent the velocity components in the $x$ and $y$ directions, respectively (i.e., the zonal and meridional wind components), we may write

$$
\begin{aligned}
& V_{T}=-u \sin \phi+v \cos \phi \\
& V_{R}=u \cos \phi+v \sin \phi
\end{aligned}
$$

where $V_{T}$ and $V_{R}$ are, respectively, the velocity components tangential to a given ellipse and radially outward from that ellipse's center. Alternately, we may express $u$ and $v$ in terms of $V_{T}$ and $V_{R}$ as

$$
\begin{gathered}
u=V_{R} \cos \phi-V_{T} \sin \phi \\
v=V_{R} \sin \phi+V_{T} \cos \phi
\end{gathered}
$$

Throughout this paper we have made the first-order assumption that

$$
\begin{aligned}
& V_{R} \equiv 0 \\
& V_{T} \equiv V_{T}(a)
\end{aligned}
$$

i.e., $V_{T}$ is a function of $a$ only. Thus,

$$
\begin{aligned}
& u \simeq-V_{T} \sin \phi \\
& v \simeq V_{T} \cos \phi
\end{aligned}
$$

In Cartesian coordinates the vertical component of vorticity is given by,

$$
\zeta=\frac{\partial v}{\partial x}-\frac{\partial u}{\partial y}
$$

Thus,

$$
\begin{aligned}
\zeta(a, x, y) & =\frac{\partial}{\partial x}\left[V_{T} \frac{x}{\left(x^{2}+\lambda^{2} y^{2}\right)^{1 / 2}}\right]+\frac{\partial}{\partial y}\left[V_{T} \frac{\lambda y}{\left(x^{2}+\lambda^{2} y^{2}\right)^{1 / 2}}\right] \\
& =V_{T} \frac{\lambda x^{2}+\lambda^{2} y^{2}}{\left(x^{2}+\lambda^{2} y^{2}\right)^{3 / 2}}+\frac{d V_{T}}{d a} \frac{\left(x^{2}+\lambda^{2} y^{2}\right)^{1 / 2}}{a}
\end{aligned}
$$

Or we may make the simple substitutions

$$
\begin{gathered}
a^{2}=x^{2}+\lambda y^{2} \\
x=r \cos \theta \\
y=r \sin \theta
\end{gathered}
$$

in order to write $\zeta$ in terms of only $a$ and $\theta$ as

$$
\begin{aligned}
\zeta(a, \theta) & =V_{T}\left[\frac{\lambda \cos ^{2} \theta+\lambda^{2} \sin ^{2} \theta}{r\left(\cos ^{2} \theta+\lambda^{2} \sin ^{2} \theta\right)^{3 / 2}}\right] \\
& +\frac{d V_{T}}{d a}\left[\frac{\cos ^{2} \theta+\lambda^{2} \sin ^{2} \theta}{\cos ^{2} \theta+\lambda \sin ^{2} \theta}\right]^{1 / 2} \\
& =\lambda V_{T}\left[\frac{1}{r^{2} \cos ^{2} \theta+r^{2} \lambda \sin ^{2} \theta}\right]^{1 / 2}\left[\frac{\cos ^{2} \theta+\lambda \sin ^{2} \theta}{\cos ^{2} \theta+\lambda^{2} \sin ^{2} \theta}\right]^{3 / 2} \\
& +\frac{d V_{T}}{d a}\left[\frac{\cos ^{2} \theta+\lambda^{2} \sin ^{2} \theta}{\cos ^{2} \theta+\lambda \sin ^{2} \theta}\right]^{1 / 2}
\end{aligned}
$$

Finally,

$$
\zeta(a, \theta)=\frac{a}{b^{2} \eta^{3}} V_{T}+\eta \frac{d V_{T}}{d a}
$$

Where

$$
\eta \equiv\left[\frac{\cos ^{2} \theta+(a / b)^{4} \sin ^{2} \theta}{\cos ^{2} \theta+(a / b)^{2} \sin ^{2} \theta}\right]^{1 / 2}
$$

Acknowledgments. We thank G. M. Yagi and P. L. Jepsen at the JPL Image Processing Laboratory for their assistance with the interactive analysis of the Voyager images and $\mathrm{C}$. J. Hansen for the mission design activities responsible for the data set we have used in this report. We also thank J. P. Müller and G. E. Hunt at the Laboratory for Planetary Atmospheres, University College London for their suggestions and comments. The research described in this paper was carried out at the Jet Propulsion Laboratory, California Institute of Technology, under contract with the National Aeronautics and Space Administration. 


\section{REFERENCES}

Beebe, R. F., A. P. Ingersoll, G. E. Hunt, J. L. Mitchell, and J.-P. Müller, Measurements of wind vectors, eddy momentum transports, and energy conversions in Jupiter's atmosphere from Voyager 1 images, Geophy. Res. Lett., 7, 1-4, 1980.

Conrath, B. J., F. M. Flasar, J. A. Pirraglia, P. J. Gierasch, and G. E. Hunt, Thermal structure and dynamics of the jovian atmosphere, 2, Visible cloud features, J. Geophys. Res., this Issue.

Flasar, F. M., B. J. Conrath, J. A. Pirraglia, P. Coark, R. French, and $P$. Gierasch, Thermal structure and dynamics of the jovian atmosphere, 1, The Great Red Spot, J. Geophys. Res., this Issue.

Hanel, R., B. Conrath, M. Flasar, V. Kunde, P. Lowman, W. Maguire, J. Pearl, J. Pirraglia, R. Samuelson, D. Gautier, P. Gierasch, S. Kumar, and C. Ponnamperuma, Infrared observations of the jovian system from Voyager 1, Science, 204, 972-976, 1979a.

Hanel, R., B. Conrath, M. Flasar, L. Herath, V Kunde, P. Lowman, W. Maguire, J. Pearl, J. Pirraglia, and C. Ponnamperuma, Infrared observations of the jovian system from Voyager 2, Science, 206, 952-956, $1979 b$.

Hess, S. L., Vorticity, Rossby number, and geostrophy in the atmosphere of Jupiter, Icarus, 11, 218-219, 1969.

Hide, R., and A. Ibbetson, An experimental study of 'Taylor columns' Icarus, S, 279-290, 1966.

Ingersoll, A. P., Inertial Taylor columns and Jupiter's Great Red Spot, J. Atmos. Sci., 26, 744-752, 1969.

Ingersoll, A. P., Jupiter's Great Red Spot: A free atmospheric vortex?, Science, 182, 1346-1348, 1973.

Ingersoll, A. P., R. F. Beebe, S. A. Collins, G. E. Hunt, J. L. Mitchell, J.-P. Muller, B. A. Smith, and R. J. Terrile, Zonal velocity and texture in the jovian atmosphere inferred from Voyager images, $\mathrm{Na}$ ture, 280, 773-775, 1979.

Ingersoll, A. P., R. F. Beebe, J. L. Mitchell, G. W. Garneau, G. M. Yagi, and J.-P. Müller, Interactions of eddies and mean zonal flow on Jupiter as inferred from Voyager 1 and 2 images, J. Geophy. Res., this Issue.

Khare, B. N., and C. Sagan, Red clouds in reducing atmospheres, Icarus, 20, 311-32!, 1973.

Maxworthy, T., and L. G. Redekopp, A solitary wave theory of the Great Red Spot and other observed features in the jovian atmosphere, Icarus, 29, 261-271, 1976.

Mitchell, J. L., R. J. Terrile, B. A. Smith, J.-P. Müller, A. P. Ingersoll, G. E. Hunt, S. A. Collins, and R. F. Beebe, Jovian cloud structure and velocity fields, Nature, 280, 776-778, 1979.

Peek, B. M., The Planet Jupiter, Faber and Faber, London, 1958.

Reese, E. J., and B. A. Smith, Evidence of vorticity in the Great Red Spot of Jupiter, Icarus, 9, 474-486, 1968.

Sagan, C., A truth table analysis of models of Jupiter's Great Red Spot, Comm. Astrophys. Space Phys., 3, 65-72, 1971.

Smith, B. A., L. A. Soderblom, R. F. Beebe, J. Boyce, G. Briggs, M. Carr, S. A. Collins, A. F. Cook, G. E. Danielson, M. E. Davies, G. E. Hunt, A. P. Ingersoll, T. V. Johnson, H. Masursky, J. McCauley, D. Morrison, T. Owen, C. Sagan, E. M. Shoemaker, R. Strom, V. E. Suomi, and J. Veverka, The Galilean satellites and Jupiter: Voyager 2 imaging science results, Science, 206, 927-950, 1979.

Streett, W. B., H. I. Ringermacher, and G. Veronis, On the structure and motions of Jupiter's Red Spot, Icarus, 14, 319-342, 1971.

Williams, G. P., Planetary circulations, 2, The jovian quasi-geostrophic regime, J. Atmos. Sci., 36, 932-968, 1979.

Yagi, G., J. Lorre, and P. Jepsen, Dynamic feature analysis for Voyager at the Image Processing Laboratory, in Proceedings of the Conference on Atmospheric Environment of Aerospace Systems and Applied Meteorology. American Meteorological Society, Boston, Mass., 1978.

(Received June 19, 1980; revised March 12, 1981; accepted March 12, 1981.) 